\title{
The effect of heat addition on slightly compressible flow: The example of vortex pair motion
}

\author{
D. W. Moore \\ Department of Mathematics, Imperial College, London, England \\ D. I. Pullin \\ Department of Mechanical Engineering, University of Queensland, Queensland, Australia
}

(Received 17 April 1990; accepted 20 March 1991)

\begin{abstract}
The effect of a point heat source on the two-dimensional irrotational flow of a slightly compressible nonviscous and nondiffusive fluid is studied. Heat is added at a constant rate and with a prescribed spatial distribution. The Mach number $M$ is assumed small and the governing equations are solved to order $M^{2}$ by a generalization of the Rayleigh-Janzen expansion. The interaction between entropy variations produced by the heat source and the pressure field generates a wake consisting of a vortex sheet and a dipole sheet extending downstream of the heat source, and this in turn perturbs the flow at order $M^{2}$. A general result is that if heat is added where the gas is compressed by the flow, the impulse directed against the free stream is increased and vice versa. Cross-stream impulse is produced if the heat source is not on the axis of symmetry (if any) of the flow. This would result in a lifting force if a cylinder is providing the perturbation to the free stream. When heat is added on the downstream dividing streamline of a symmetrical flow the order $M^{2}$ velocity field at infinity is that due to an apparent mass source and it is shown that the required flux of mass and momentum is supplied from infinity via the wake. Detailed calculations are performed for the case of the self-propagation of a symmetrical vortex pair, where heat is added downstream of the rearward stagnation point. The resultant increase in the pair impulse is shown to lead to a slow increase in the vortex pair separation.
\end{abstract}

\section{INTRODUCTION}

Recent work (Karagozian and Manda ${ }^{1}$ and the references cited therein) has shown that vortical flows can drastically modify combustion. This is because the presence of vorticity produces stretching of the interface between fuel and oxidizer thus providing a much increased interfacial area across which fuel-oxidizer mixing, and hence combustion, can occur. Karagozian and Manda studied the effects of a vortex pair on a fuel strip, each point vortex lying on a strip boundary. The deformation of the pre-existing flame by the flow was calculated and found to be significant. In their analysis Karagozian and Manda assume the flow to be inviscid and incompressible, and it follows that the heat released in the combustion process at the strip-fluid interface can in no way modify the flow structure: any heat will behave purely as a convected passive scalar.

In real combusting flows it is to be expected that substantial heat release will perturb the flow structure via gas compressibility. Numerical solution of the equations of a compressible, heat conducting gas in which combustion is taking place is possible (McMurtry et al. ${ }^{2}$ ), provided that sound waves are filtered, but our aim is to study the basic mechanism that drives this interaction and to illustrate this in a highly simplified case for which analytical treatment is straightforward. We ignore the detailed physics of the combustion process and simply specify the rate of release as a function of position. This approach, for two-dimensional flow, is due to Broadbent. ${ }^{3}$ A general class of exact solutions of the Euler equations with heat addition was constructed by Chenoweth, ${ }^{4}$ but the heat addition could not be a prescribed function of position.

In Sec. II we consider the fundamental problem consisting of the effect of a point heat source on the two-dimensional flow of an inviscid and slightly compressible fluid. The governing equations are expanded to order $M^{2}$, where $M$ is an appropriately defined Mach number. It is clear that the approximate equations capture the basic physics, namely the production of entropy by the heat addition [Eq. (25)] and the consequent production of vorticity by the interaction between the pressure and entropy fields [Eq. (33)].

At order $M^{2}$, the vorticity and entropy convect along streamlines of the incompressible or zeroth-order flow so that the calculation of the distribution of these quantities is helped by making the Boussinesq transformation, in which the velocity potential and streamfunction of the incompressible flow are employed as coordinates. This calculation is carried out in Sec. III for the case of a point heat source on an arbitrary but open streamline of the incompressible flow. The result is that the entropy and vorticity are distributed on that part of the streamline extending downstream of the heat source to infinity: the vorticity distribution consists of a vortex sheet combined with a dipole sheet.

At this stage the analysis is quite general, but in Sec. IV we specialize first to the case of a point heat source on the dividing (open) streamline of a symmetrical flow. This leads 
to an integral expression for the order $M^{2}$ perturbation velocity field in terms of the zeroth-order flow [Eq. (59)] from which we can deduce that the far field of the vortex wake is sourcelike.

In order to illustrate the present results we then select for detailed study the flow due to a vortex pair propagating in an unbounded fluid. For the isentropic case (i.e., no heat addition) this flow has been treated numerically and analytically by Moore and Pullin ${ }^{5}$ - henceforth referenced as MP, who found that the $M^{2}$ term in the Rayleigh-Janzen expansion, where $M$ is the Mach number based on the propagation velocity, gave core shapes that agreed well with numerical results obtained using a hodograph technique. In a reference frame moving with the pair, the zeroth-order flow has a pair of stagnation points so that the dividing streamline bounds a region $\mathbf{R}$ of closed streamlines (Fig. 1). We imagine that this region contains fuel that diffuses across the bounding streamline to react with oxidizer contained in the exterior flow. We assume that heat will be released outside the region $\mathbf{R}$, perhaps some distance downstream if the reaction is suffciently slow. Since we are neglecting diffusion effects in our detailed calculations, we must appeal to convection by the flow to maintain a steady temperature distribution. Thus we cannot deal with heat released in the interior of $\mathbf{R}$, where a diffusive analysis (Krönig and Brink ${ }^{6}$ and Brignell ${ }^{7}$ ) is essential. Since the supply of fuel is finite, the flow cannot be strictly steady, but we can consistently neglect this when diffusion is small.

Under these assumptions the effect of the point heat source is calculated explicitly and the most striking effect is that the diameter of the pair slowly increases with comparable contributions to the expansion velocity from both the apparent mass source and the wake vorticity distributions. (The resulting unsteadiness affects the dynamics at order $M^{4}$.) This implies an input of momentum to the flow that

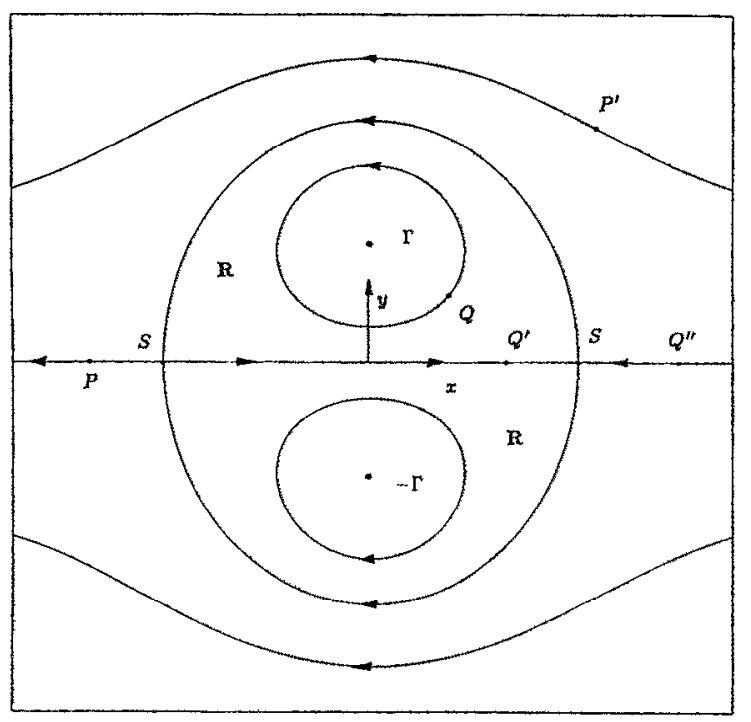

FIG. 1. A sketch of the streamlines of the flow past a stationary vortex pair. We can deal with a heat source at points $P_{2} P^{\prime}$ but not at $Q, Q^{\prime}$, or $Q^{\prime \prime}$. The point vortices are at $(0, \pm 1)$ and the stagnation points $S$ at $( \pm v 3,0)$. can only be supplied by an influx of momentum along the wake created by the heat source.

This is confirmed in Sec. $V$ where the detailed mass and momentum balances are investigated in a general setting, and illustrated for the vortex pair flow. For a heat source located on an open streamline in a general steady flow it is shown that momentum opposed to the free stream is added provided the fluid is compressed at the heat source [Eq. (86) ]. In addition, net vorticity is produced and the concomitant circulation leads to a lifting force if a cylinder of arbitrary cross-sectional shape is providing the flow perturbation.

\section{THE GOVERNING EQUATIONS}

We choose axes $0 x^{\prime} y^{\prime}$ and consider, at this stage, a general two-dimensional inviscid incompressible and irrotational flow characterized by a velocity scale $U_{\infty}$ and a length scale $L$. Later we will consider the particular example of the vortex pair flow for which $0 x^{\prime} y^{\prime}$ are fixed in the vortex pair, such that the point vortex of circulation $\Gamma$ is at $(0, L)$ and that of circulation $-\Gamma$ is at $(0,-L)$. In this case there is at infinity a flow velocity $\left(-U_{\infty}, 0\right)$ where, if compressibility is neglected,

$$
U_{\infty}=\Gamma / 4 \pi L
$$

the correction to this formula introduced by slight compressibility was calculated in MP.

The Euler equations for steady motion are

$$
\begin{aligned}
& u^{\prime} \frac{\partial u^{\prime}}{\partial x^{\prime}}+v^{\prime} \frac{\partial u^{\prime}}{\partial y^{\prime}}=-\frac{1}{\rho^{\prime}} \frac{\partial p^{\prime}}{\partial x^{\prime}}, \\
& u^{\prime} \frac{\partial v^{\prime}}{\partial x^{\prime}}+v^{\prime} \frac{\partial v^{\prime}}{\partial y^{\prime}}=-\frac{1}{\rho^{\prime}} \frac{\partial p^{\prime}}{\partial y^{\prime}}, \\
& \frac{\partial}{\partial x^{\prime}}\left(\rho^{\prime} u^{\prime}\right)+\frac{\partial}{\partial y^{\prime}}\left(\rho^{\prime} v^{\prime}\right)=0, \\
& u^{\prime} \frac{\partial s^{\prime}}{\partial x^{\prime}}+v^{\prime} \frac{\partial s^{\prime}}{\partial y^{\prime}}=\frac{q^{\prime}\left(x^{\prime}, y^{\prime}\right)}{T^{\prime}} .
\end{aligned}
$$

In these equations the prime denotes that the flow variables are in physical units and $\left(u^{\prime}, v^{\prime}\right)$ is the velocity field, $p^{\prime}$ is the pressure, $\rho^{\prime}$ is the density, $s^{\prime}$ is the specific entropy, and $T^{\prime}$ is the absolute temperature. Here, $q^{\prime}\left(x^{\prime}, y^{\prime}\right)$ is a prescribed heat addition per unit mass per unit time. We assume a calorically perfect gas, so that the entropy equation of state can be written as

$$
s^{\prime}-s_{\infty}=c_{v} \ln \left(p^{\prime} / p_{\infty}\right)-c_{p} \ln \left(\rho^{\prime} / \rho_{x}\right),
$$

where $c_{v}, c_{p}$ are the constant specific heats and $p_{*}, \rho_{\infty}$, and $s_{\infty}$ are the values of pressure, density, and specific entropy at infinity, respectively.

We introduce dimensionless flow variables via the scalings

$$
\begin{aligned}
& \left(u^{\prime}, v^{\prime}\right)=U_{\infty}(u, v), \\
& \left(x^{\prime}, y^{\prime}\right)=L(x, y), \\
& p^{\prime}=p_{\infty} p,
\end{aligned}
$$

and

$$
\rho^{\prime}=\rho_{x} \rho
$$


we consider the scalings appropriate to the remaining thermodynamic variables later.

Equations (2)-(4) become

$$
\begin{aligned}
& M^{2}\left(u \frac{\partial u}{\partial x}+v \frac{\partial u}{\partial y}\right)=-\frac{1}{\gamma \rho} \frac{\partial p}{\partial x} \\
& M^{2}\left(u \frac{\partial v}{\partial x}+v \frac{\partial v}{\partial y}\right)=-\frac{1}{\gamma \rho} \frac{\partial p}{\partial y}
\end{aligned}
$$

and

$$
\frac{\partial}{\partial x}(\rho u)+\frac{\partial}{\partial y}(\rho v)=0
$$

Here $M^{2}=U_{\infty}^{2} / C_{\infty}^{2}$, where $C_{\infty}^{2}=\gamma p_{\infty} / \rho_{\infty}$ and where $\gamma=c_{p} / c_{v}$, so that $C_{\infty}$ is the speed of sound at $\infty$.

The form of Eqs. (8)-(10) suggests the expansions

$$
\begin{aligned}
& u=u_{0}+M^{2} u_{1}+\cdots, \\
& v=v_{0}+M^{2} v_{1}+\cdots, \\
& p=1+M^{2} p_{1}+\cdots,
\end{aligned}
$$

and

$$
\rho=1+M^{2} \rho_{1}+\cdots .
$$

Here $\left(u_{0}, v_{0}\right)$ is the velocity field of the irrotational incompressible fluid that will be modified by compressibility and heat addition effects, while $p_{1}$ is the pressure variation associated with $\left(u_{0}, v_{0}\right)$. Using (11)-(14) in (8) and (9) then gives

$$
p_{1}=\frac{1}{2} \gamma\left(1-u_{0}^{2}-v_{0}^{2}\right) .
$$

We remark that it is the scaling of the pressure with $p_{\infty}$ (instead of the customary $\rho_{\infty} U_{\infty}^{2}$ ) that makes the pressure variations associated with the basic irrotational flow appear as an $O\left(M^{2}\right)$ correction. This scaling reflects the fact that these variations are small when viewed from a thermodynamic standpoint.

Next we substitute (11), (12), and (14) into the equation of continuity $(10)$ to find that

$$
\frac{\partial u_{1}}{\partial x}+\frac{\partial v_{1}}{\partial y}+u_{0} \frac{\partial \rho_{1}}{\partial x}+v_{0} \frac{\partial \rho_{1}}{\partial y}=0
$$

We wish to avoid the computation of $p_{2}$, the compressibility correction to the pressure, so we turn to the vorticity equation. In dimensionless form, this is

$$
\begin{gathered}
M^{2}\left[u \frac{\partial \omega}{\partial x}+v \frac{\partial \omega}{\partial y}+\omega\left(\frac{\partial u}{\partial x}+\frac{\partial v}{\partial y}\right)\right] \\
=\frac{1}{\gamma \rho^{2}}\left(\frac{\partial \rho}{\partial x} \frac{\partial p}{\partial y}-\frac{\partial \rho}{\partial y} \frac{\partial p}{\partial x}\right),
\end{gathered}
$$

where we have scaled the physical vorticity $\omega^{\prime}(x, y)$ by

$$
\omega^{\prime}=\left(U_{\infty} / L\right) \omega .
$$

We now expand the vorticity in powers of $M^{2}$, so that

$$
\omega=M^{2} \omega_{1}+\cdots
$$

and substitute into (17) to find that

$u_{0} \frac{\partial \omega_{1}}{\partial x}+v_{0} \frac{\partial \omega_{1}}{\partial y}=\frac{1}{\gamma}\left(\frac{\partial \rho_{1}}{\partial x} \frac{\partial p_{1}}{\partial y}-\frac{\partial \rho_{1}}{\partial y} \frac{\partial p_{1}}{\partial x}\right)$.

In the absence of entropy variations, the right-hand side of (20) would vanish and then $\omega_{1}$ would be constant on the streamlines of the irrotational flow. Thus to compute the production of vorticity, we must next consider the variation of entropy. Let $e_{\infty}$ be the specific internal energy of the incoming fluid at infinity and $T_{\infty}$ its absolute temperature. Then $e_{\infty}=c_{v} T_{\infty}$ and a convenient scaling for the entropy is

$$
s^{\prime}-s_{\infty}=\left(e_{\infty} / T_{\infty}\right) s,
$$

where

$$
s=M^{2} s_{1}+\cdots .
$$

Then the equation of state (6) together with (11)-(14) yields the linearized equation of state

$$
s_{1}=p_{1}-\gamma \rho_{1} .
$$

Finally, we introduce a dimensionless rate of heat addition $q_{1}$ defined so that

$$
q^{\prime}\left(x^{\prime}, y^{\prime}\right)=\left(U_{\infty} e_{\infty} / L\right) M^{2} q_{1}(x, y) .
$$

Evidently the temperature variations in the entropy production equation (5) are negligible to leading order, so that it reduces to

$$
u_{0} \frac{\partial s_{1}}{\partial x}+v_{0} \frac{\partial s_{1}}{\partial y}=q_{1} .
$$

To solve Eqs. (16), (20), and (25) we introduce potentials $\phi_{1}$ and $\psi_{1}$ such that

$$
\begin{aligned}
& u_{1}=\frac{\partial \phi_{1}}{\partial x}+\frac{\partial \psi_{1}}{\partial y} \\
& v_{1}=\frac{\partial \phi_{1}}{\partial y}-\frac{\partial \psi_{1}}{\partial x}
\end{aligned}
$$

Substituting in the continuity equation (16) and using the linearized equation of state (23) gives

$\gamma \nabla^{2} \phi_{1}=-u_{0} \frac{\partial p_{1}}{\partial x}-v_{0} \frac{\partial p_{1}}{\partial y}+u_{0} \frac{\partial s_{1}}{\partial x}+v_{0} \frac{\partial s_{1}}{\partial y}$,

or in view of (25),

$\gamma \nabla^{2} \phi_{1}=-u_{0} \frac{\partial p_{1}}{\partial x}-v_{0} \frac{\partial p_{1}}{\partial y}+q_{1}$.

Equation (28) was derived by Broadbent, ${ }^{8}$ who pointed out that the region of heat addition behaves as a source of mass. Wc will sec later that this mass flux is provided by a wake extending from the region of heat addition to downstream infinity. There is, of course, no true mass source, as is apparent from the equation of continuity (10). We can split the problem further by writing

$$
\phi_{1}=\phi_{1 R}+\phi_{1 H}
$$

and choosing

$$
\nabla^{2} \phi_{1 H}=q_{1} / \gamma
$$

and

$$
\nabla^{2} \phi_{1 R}=\frac{1}{2}\left(u_{0} \frac{\partial}{\partial x}+v_{0} \frac{\partial}{\partial y}\right)\left(u_{0}^{2}+v_{0}^{2}\right)
$$

on using (15) to eliminate the pressure $p_{1}$. Now

$$
\nabla^{2} \psi_{1}=-\omega_{1}
$$

so the next step is to determine the vorticity field. We eliminate $\rho_{1}$ from the vorticity equation (20) by using (23) to find 


$$
u_{o} \frac{\partial \omega_{1}}{\partial x}+v_{o} \frac{\partial \omega_{1}}{\partial y}=\frac{1}{\gamma^{2}} \frac{\partial\left(p_{1}, s_{1}\right)}{\partial(x, y)} .
$$

Equations (30) and (31) serve to determine $\phi_{1}$ while (15), (25), (32), and (33) likewise serve to determine $\psi_{1}$. We discuss the imposition of appropriate boundary conditions in the next section.

We note one special case. If we set $q_{1}=0$, then (25) shows that we can consistently set $s_{1}=0$ and then (33) shows we can take $\omega_{1}=0$. Then $\phi_{1 H}=\psi_{1}=0$ and the problem collapses to solving $\mathrm{Eq}$. (31), which emerges as the governing equation for the second term of the RayleighJanzen expansion. As first discussed by Barsony-Nagy et $a l .{ }^{9}$ this expansion fails near point vortices in the flow and recourse to the method of matched asymptotic expansions is necessary. The details for the special case of a vortex pair are given in MP and thus we need not consider $\phi_{1 R}$ further.

\section{THE DETERMINATION OF THE ENTROPY AND VORTICITY FIELDS}

We shall consider the case of a single point source of heat at the position $(\bar{x}, \bar{y})$, so that

$$
q_{1}(x, y)=q \delta(x-\bar{x}) \delta(y-\bar{y}) .
$$

Since the governing equations are linear, the resulting solution for the potentials $\phi_{1 H}$ and $\psi_{1}$ will act as a Green's function.

It is convenient to replace $(x, y)$ by $(\phi, \psi)$, the velocity potential and streamfunction of the unperturbed flow, defined by

$$
u_{0}=\frac{\partial \phi}{\partial x}=\frac{\partial \psi}{\partial y}
$$

and

$$
v_{0}=\frac{\partial \phi}{\partial y}=-\frac{\partial \psi}{\partial x}
$$

It follows that

$$
\frac{\partial(\phi, \psi)}{\partial(x, y)}=J(\phi, \psi)=u_{0}^{2}+v_{0}^{2},
$$

so that the transformation is singular both at the stagnation points and, in the case of the vortex pair, at the point vortices themselves. The entropy equation (25) becomes

$$
J(\phi, \psi) \frac{\partial s_{1}}{\partial \phi}=q J(\bar{\phi}, \bar{\psi}) \delta(\phi-\bar{\phi}) \delta(\psi-\bar{\psi})
$$

or, equivalently,

$$
\frac{\partial s_{1}}{\partial \phi}=q \delta(\phi-\bar{\phi}) \delta(\psi-\bar{\psi}) .
$$

It is clear that if the point $(\bar{\phi}, \bar{\psi})$ lies on a closed streamline an inconsistency arises, because Eq. (38) gives, on integration,

$$
s_{1}\left(\phi_{0}, \bar{\psi}\right)=s_{1}\left(\phi_{0}+\Gamma, \bar{\psi}\right)+q,
$$

where $\Gamma$ is the circulation around the family of closed streamlines of which $\bar{\psi}$ is a member and $\phi_{0} \neq \bar{\phi}$, but is otherwise arbitrary. Since $\phi_{0}, \bar{\psi}$ and $\phi_{0}+\Gamma, \bar{\psi}$ correspond to the same $(x, y),(39)$ shows that the entropy cannot be a single valued function. This inconsistency is a consequence of the neglect of diffusion. Heat cannot escape from a region of closed streamlines by convection which, as shown in Ref. 5 , serves only to make the temperature distribution independent of $\phi$ when the Péclet number is sufficiently large.

No difficulty arises on a streamline going from upstream infinity $(\phi \rightarrow-\infty)$ to downstream infinity $(\phi \rightarrow \infty)$ without passing through a stagnation point. We can now see that the appropriate conditions that determine $s_{1}$ and $\omega_{1}$ are

$$
s_{1}=\omega_{1}=0 \text { as } x \rightarrow \infty, \text { all } y
$$

(see Fig. 1). It follows from the entropy equation (38) that

$$
s_{1}=q H(\phi-\bar{\phi}) \delta(\psi-\bar{\psi}),
$$

where $H$ is the Heaviside function.

The vorticity equation (33) transforms to

$$
\frac{\partial \omega_{1}}{\partial \phi}=\frac{1}{\gamma^{2}} \frac{\partial\left(p_{1}, s_{1}\right)}{\partial(\phi, \psi)}
$$

and from Bernoulli's equation (15)

$$
p_{1}=\frac{1}{2} \gamma[1-J(\phi, \psi)],
$$

so that, in view of (41) and (43)

$$
\begin{aligned}
\frac{\partial \omega_{1}}{\partial \phi}= & -\frac{q}{2 \gamma}\left(\frac{\partial J}{\partial \phi} H(\phi-\bar{\phi}) \delta^{\prime}(\psi-\bar{\psi})\right. \\
& \left.-\frac{\partial J}{\partial \psi} \delta(\phi-\bar{\phi}) \delta(\psi-\bar{\psi})\right) .
\end{aligned}
$$

Thus integrating and invoking the boundary condition (37),

$$
\begin{aligned}
\omega_{1}= & \frac{q}{2 \gamma} H(\phi-\bar{\phi})\left[(J(\bar{\phi}, \psi)-J(\phi, \psi)) \delta^{\prime}(\psi-\bar{\psi})\right. \\
& \left.+\frac{\partial J}{\partial \psi}(\bar{\phi}, \psi) \delta(\psi-\bar{\psi})\right] .
\end{aligned}
$$

Equations (41) and (45) show that the entropy and vorticity are confined to a singular wake on the streamline $\psi=\bar{\psi}$ downstream of the heat source at the point $(\bar{\phi}, \bar{\psi})$. The vorticity field (45) takes the form of a dipole sheet, given by the first term in square brackets and a vortex sheet represented by the second term in square brackets, each extending to infinity downstream of $(\bar{\phi}, \bar{\psi})$.

Equation (30) becomes

$$
\nabla^{2} \phi_{1 H}=(q / \gamma) \delta(x-\bar{x}) \delta(y-\bar{y}),
$$

whose solution is

$$
\phi_{1 H}=(q / 4 \pi \gamma) \ln \left[(x-\bar{x})^{2}+(y-\bar{y})^{2}\right] .
$$

Using (2) and (45) we can write down a formal solution for $\psi_{1}$, but the result is cumbersorne. Moreover, we can further develop the form of the entropy wake when there is a small but finite diffusivity but the result is complicated and the spirit of the present study is to elucidate the the order $M^{2}$ perturbation to the inviscid global flow. We prefer to illustrate the effect of heat addition on the flow by examining in detail the special and simplified case of a point source of heat on the downstream axis of symmetry of the flow past a stationary vortex pair. This is studied in Sec. IV.

Finally we note that $\nabla \phi_{1 H} \rightarrow \infty$ as $(x, y) \rightarrow(\bar{x}, \bar{y})$, so that the expansions (11) and (12) become invalid. However, in reality, the solution of the problem defined by the point source of heat (44) is merely a Green's function for the solu- 
tion for an analytic distribution $q_{1}(x, y)$ and in this case there is no difficulty with the linearization.

\section{THE EFFECT OF HEAT ADDITION ON A VORTEX PAIR}

We now restrict attention to flows without solid boundaries and with mirror image symmetry in the streamline pattern. Further the heat source modeling combustion is supposed to be located at $(\bar{x}, 0)$ on the dividing streamline $y=0$, which extends downstream to $x \rightarrow \infty$. We will later consider explicitly the vortex pair example.

The last term in Eq. (45) then vanishes, because

$$
\frac{\partial J}{\partial \psi}=2 u_{0} \frac{\partial u_{0}}{\partial \psi}+2 v_{0} \frac{\partial v_{0}}{\partial \psi}=0 \text { on } \psi=0
$$

since $v_{0}=0$ and $\partial u_{0} / \partial \psi=0$ on $\psi=0$, by symmetry. Thus, for $\phi \geqslant \bar{\phi}$, the vortex sheet term vanishes and we are left with the dipole sheet given by

$$
\omega_{1}(\phi, \psi)=(q / 2 \gamma)[J(\bar{\phi}, \psi)-J(\phi, \psi)] \delta^{\prime}(\psi) .
$$

Since there are no solid boundaries, the $O\left(M^{2}\right)$ complex velocity $V_{1 \omega}$ derived from $\psi_{1}$ at the point $\hat{z}$, not on the wake, is given by

$$
\begin{aligned}
V_{1 \omega}(\hat{z})= & -\frac{i q}{4 \pi \gamma} \int_{\bar{\phi}}^{\infty} d \phi \\
& \times \int_{-\epsilon}^{\epsilon} \frac{[J(\bar{\phi}, \psi)-J(\phi, \psi)] \delta^{\prime}(\psi) d \psi}{[\hat{z}-z(\phi, \psi)] J(\phi, \psi)},
\end{aligned}
$$

where $z(\phi, \psi)$ is complex position expressed as a function of $\phi$ and $\psi$ and where $\epsilon$ is any small positive number. To evaluate the inner integral, we use integration by parts to find that

$$
V_{1(a)}=\frac{i q}{4 \pi \gamma} \int_{\bar{\phi}}^{\infty} d \phi \int_{-\epsilon}^{\epsilon} \frac{\partial A}{\partial \psi} \delta(\psi-\bar{\psi}) d \psi,
$$

where

$$
A=\frac{1}{\hat{z}-z(\phi, \psi)}\left(\frac{J(\bar{\phi}, \psi)}{J(\phi, \psi)}-1\right),
$$

so that, invoking (48) again,

$$
V_{1 \omega}=\frac{i q}{4 \pi \gamma} \int_{\bar{\phi}}^{\infty}\left(\frac{J(\bar{\phi}, 0)}{J(\phi, 0)}-1\right) \frac{(\partial z / \partial \psi)(\phi, 0) d \phi}{[\hat{z}-x(\phi, 0)]^{2}} .
$$

Now

$$
\frac{\partial z}{\partial \psi}=i \frac{\partial z}{\partial w_{0}}=\frac{i}{u_{0}-i v_{0}},
$$

where $w_{0}(z)$ is the complex potential of the irrotational flow $\left(u_{0}, v_{0}\right)$, so that, finally,

$$
V_{1 \kappa \nu}(\hat{z})=\frac{q}{4 \pi \gamma} \int_{-\infty}^{\bar{x}}\left(\frac{u_{0}^{2}(\bar{x})}{u_{0}^{2}(x)}-1\right) \frac{d x}{(\hat{z}-x)^{2}},
$$

where (36) has been used.

In the subsequent section we examine the far field induced by the heat source and for this purpose we write (55) in the form

$$
\begin{aligned}
V_{1 \omega}(\hat{z})= & \frac{q}{4 \pi \gamma} \int_{-\infty}^{\bar{x}}\left[\left(\frac{u_{0}^{2}(\bar{x})}{u_{0}^{2}(x)}-u_{0}^{2}(\bar{x})\right)\right. \\
& \left.+\left[u_{0}^{2}(\bar{x})-1\right]\right] \frac{d x}{(\hat{z}-x)^{2}}
\end{aligned}
$$

so that

$$
\begin{aligned}
V_{1 \omega}(\hat{z})= & \frac{q}{4 \pi \gamma} u_{0}^{2}(\bar{x}) \int_{-\infty}^{\bar{x}}\left(\frac{1}{u_{0}^{2}(x)}-1\right) \frac{d x}{(\hat{z}-x)^{2}} \\
& +\frac{q}{4 \pi \gamma} \frac{\left[u_{0}^{2}(\bar{x})-1\right]}{(\hat{z}-\bar{x})} .
\end{aligned}
$$

Thus the far field of the flow induced by the vortex wakc corresponds to a source. If $V_{1 H}(\hat{z})$ is the complex velocity corresponding to $\phi_{1 H}$ given in Eq. (47), then

$$
V_{1 H}(\hat{z})=(q / 2 \pi \gamma)[1 /(\hat{z}-\bar{x})]
$$

and defining $V_{1}=V_{1 \omega}+V_{1 H}$ to be the total complex velocity induced by the heat source,

$$
\begin{aligned}
V_{1}(\hat{z})= & \frac{q u_{0}^{2}(\bar{x})}{4 \pi \gamma} \int_{-\infty}^{\bar{x}}\left(\frac{1}{u_{0}^{2}(x)}-1\right) \frac{d x}{(\hat{z}-x)^{2}} \\
& +\frac{q}{4 \pi \gamma} \frac{\left[u_{0}^{2}(\bar{x})+1\right]}{(\hat{z}-\bar{x})} .
\end{aligned}
$$

To illustrate the effect of heat addition on a slightly compressible flow with symmetrical streamlines, we now consider specifically the example of the incompressible flow due to a vortex pair propagating in an unbounded fluid. In terms of the kinematic scaling for this flow indicated in Sec. II, the uniform stream has dimensionless velocity $(-1,0)$ at infinity (neglecting corrections due to the small stagnant cores). We consider the perturbation to the vortex pair flow when the heat source is located on the axis of symmetry downstream of the trailing stagnation point. Thus we restrict ourselves to the case $\bar{x}<-\sqrt{3}, \bar{y}=0$. The velocity on this streamline is then

$$
u_{0}(x)=\left(3-x^{2}\right) /\left(x^{2}+1\right)
$$

so that substitution into (59) enables $V_{1}(\hat{z})$ to be evaluated.

The most interesting effect is that of the thermally induced flow field on the vortex pair, so we evaluate $V_{1}(i)$ to find

$$
\begin{aligned}
V_{1}(i)= & \frac{q}{4 \pi \gamma}\left[\frac{1}{3 \sqrt{3}} \frac{\left(3-\bar{x}^{2}\right)^{2}}{\left(\bar{x}^{2}+1\right)^{2}} \ln \left(\frac{\bar{x}-\sqrt{3}}{\bar{x}+\sqrt{3}}\right)\right. \\
& \left.-\frac{4 \bar{x}^{3}}{3\left(\bar{x}^{2}+1\right)^{2}}-2 i \frac{\left(\bar{x}^{2}-1\right)}{\left(\bar{x}^{2}+1\right)^{2}}\right] .
\end{aligned}
$$

We remark that $V_{1}(i)$ may be viewed as the sum of comparable contributions from the entropy wake and from the apparent mass source, corresponding to the first and second terms of (59), respectively. The real part of $V_{1}$ yields a small modification to the translational velocity of the vortex pair which, incorporating the correction due to the Rayleigh-Janzen field $\phi_{1 R}$ calculated in MP, becomes $U$ where

$$
U=U_{\infty}\left[1+M^{2}\left(-\frac{1}{4}+\frac{q}{12 \pi \gamma} f(\bar{x})\right)\right],
$$

where 
$f(\bar{x})=\frac{1}{\sqrt{3}} \frac{\left(3-\bar{x}^{2}\right)^{2}}{\left(\bar{x}^{2}+1\right)^{2}} \ln \left(\frac{\bar{x}-\sqrt{3}}{\bar{x}+\sqrt{3}}\right)-\frac{4 \bar{x}^{3}}{\left(\bar{x}^{2}+1\right)^{2}}$

and where $U_{\infty}$ is defined in Eq. (1).

A more striking effect arises from the velocity imparted to the point vortex in the $y$ direction. This causes the distance $2 L$ between the point vortices comprising the pair to increase with time at a rate given by

$$
\frac{d}{d t}(2 L)=-2 M^{2} U_{\infty} \operatorname{Im}\left\{V_{1}(i)\right\} .
$$

Thus our assumption of steady flow is inconsistent, but it can be shown the unsteadiness alters the dynamics of the flow field only at order $M^{4}$. To see this we note that the velocity potential $L U_{\infty} \phi$ satisfies the (Woods, ${ }^{10}$ p. 33) equation

$$
\begin{aligned}
\frac{C^{2}}{C_{\infty}^{2}} \nabla^{2} \phi= & \frac{M^{2}}{2} \nabla \phi \cdot \nabla(\nabla \phi)^{2} \\
& +M^{2}\left(2 \nabla \phi \cdot \frac{\partial}{\partial t}(\nabla \phi)+\frac{\partial^{2} \phi}{\partial t^{2}}\right),
\end{aligned}
$$

where $t^{\prime}=\left(L / U_{\infty}\right) t$ and $C$ is the local sound speed. But $\partial / \partial t=O\left(M^{2}\right)$, so the result follows. This expansion of the vortex pair causes the impulse $P^{\prime}$, given by

$$
P^{\prime}=2 p_{\infty} \Gamma L
$$

to increase at a rate

$$
\frac{d P^{\prime}}{d t^{\prime}}=\frac{4 \rho_{\infty} U_{\infty}^{2} L q M^{2}\left(\bar{x}^{2}-1\right)}{\gamma\left(\bar{x}^{2}+1\right)^{2}} .
$$

Thus the heat source is adding momentum to the flow. Equation (1) shows that this, paradoxically, is associated with a slowing down of the pair. However the equations of motion (2), (3), and (4) show that there is no source of mass or momentum at the heat source itself, so that the momentum must be supplied from infinity via the wake. We examine this idea in the next section.

\section{THE MASS AND MOMENTUM FLUX BALANCES}

We consider first the mass flux and stress that the following analysis is not restricted to the vortex pair flow although for the present the source is still constrained to lie on the dividing streamline of a symmetric flow. The RayleighJanzen solution $\phi_{1 R}$ is $O\left(|\hat{z}|^{-1}\right)$ at infinity (MP) so it makes no contribution to the mass flux. The total velocity $V_{1}(\hat{z})$ induced by the heat source is, for large $|\hat{z}|$,

$$
V_{1}(\hat{z})=\frac{q}{4 \pi \gamma} \frac{\left[u_{0}^{2}(\bar{x})+1\right]}{\hat{z}}+O\left(|z|^{-2}\right),
$$

since the term involying the integral in (59) behaves like a dipole at large distances. Now the outward mass flux through a large circle of radius $L R$ is $M^{\prime}$, where

$$
M^{\prime}=L \int_{0}^{2 \pi} \rho^{\prime}\left(u^{\prime} \cos \theta+v^{\prime} \sin \theta\right) R d \theta,
$$

where $\theta$ is a polar angle measured anticlockwise from the position $x$ axis. Outside the wake, where $s_{1}=0$, we have from the linearized equation of state (23) that

$$
\rho_{1}=+p_{1} / \gamma=O\left(R^{-2}\right)
$$

where we have used (15) and the estimates

$$
u_{0}+1=O\left(R^{-2}\right)
$$

and

$$
v_{0}=O\left(R^{-2}\right) .
$$

Thus the contribution to the mass flux from the flow outside the wake $M_{e}^{\prime}$ is given by

$$
M_{e}^{\prime}=\rho_{\infty} U_{\infty} L M^{2} \int_{0}^{2 \pi}\left(u_{1} \cos \theta+v_{1} \sin \theta\right) R d \theta,
$$

so that, in view of (68),

$$
M_{e}^{\prime}=\left(\rho_{\infty} U_{\infty} L M^{2} q / 2 \gamma\right)\left[u_{0}^{2}(\bar{x})+1\right]
$$

The outward mass flux in the wake $M_{\omega}^{\prime}$ is

$$
M_{\omega 0}^{\prime}=\rho_{\infty} U_{\infty} L M^{2} \int_{-\epsilon}^{\epsilon}\left(\rho_{1}-u_{1}\right) d y
$$

Now the pressure in the wake equals that just outside, which is zero by (70). Hence, invoking the linearized equation of state (23), and using (41) with $\bar{\psi}=0$ gives

$$
\rho_{1}=-s_{1} / \gamma=-(q / \gamma) \delta(\psi)
$$

To evaluate the contribution of the second term in Eq. (74) we integrate by parts to find

$$
\int_{-\epsilon}^{\epsilon} u_{1} d y=\left(y u_{1}\right)_{-\epsilon}^{+\epsilon}+\int_{-\epsilon}^{\epsilon} y \omega_{1}(y) d y
$$

and since the integrated term is zero, we get

$$
\begin{aligned}
M_{\omega}^{\prime}= & \frac{\rho_{\infty} U_{\infty} L M^{2} q}{\gamma} \int_{-\epsilon}^{\epsilon}\left[-\delta(\psi)+\frac{1}{2} \psi(J(\bar{\phi}, \psi)\right. \\
& \left.-J(\phi, \psi)) \delta^{\prime}(\psi)\right] d \psi,
\end{aligned}
$$

where we have used (49), the relation $\psi \sim-y$ as $x \rightarrow-\infty$ and the facts that $\delta$ and $\delta^{\prime}$ are even and odd functions, respectively. Evaluating the integral, appealing to the symmetry condition (48) gives

$$
M_{\omega}^{\prime}=\left(\rho_{\infty} U_{\infty} L M^{2} q / \gamma\right)\left\{-1-\frac{1}{2}[J(\bar{\phi}, 0)-J(\infty, 0)]\right\}
$$

so that in view of (36),

$M_{\alpha}^{\prime}=-\left(\rho_{\infty} U_{\infty} L M^{2} q / 2 \gamma\right)\left[u_{0}^{2}(\bar{x})+1\right]$.

Thus

$M_{e}^{\prime}+M_{\omega}^{\prime}=0$

confirming that the wake influx provides all the mass needed to supply the source evident in Eq. (68).

We now consider the influx at momentum $F^{\prime}$ in the wake, which is (Ref. 10, p. 19),

$$
F^{\prime}=\rho_{x} U_{x}^{2} L M^{2} \int_{-\epsilon}^{\epsilon} u_{0} u_{1} d y .
$$

We have alrcady performed the necessary integration and

$$
F^{\prime}=-\left(\rho_{\infty} U_{\infty}^{2} L q M^{2} / 2 \gamma\right)\left[u_{0}^{2}(\bar{x})-1\right] .
$$

For the special case of the vortex pair flow, substituting for $u_{0}(\bar{x})$ from $(60)$ gives, 


$$
F^{\prime}=\left[4 \rho_{\infty} U_{\infty}^{2} L q M^{2}\left(\bar{x}^{2}-1\right)\right] / \gamma\left(\bar{x}^{2}+1\right)^{2} .
$$

Thus, comparing (67) and (83),

$$
\frac{d P^{\prime}}{d t^{\prime}}=F^{\prime},
$$

verifying that the increase of the impulse of the vortex pair is provided by the momentum influx in the wake.

We can use the momentum flux to find the rate of increase of impulse of the fluid in the more general case of a zeroth-order incompressible flow which is not necessarily symmetric and for which the heat source is not constrained to lie on the dividing streamline. We could, for example, have flow past a cylinder of arbitrary cross section. We consider a heat source $P$ at any point $(\bar{x}, \bar{y})$ lying on an open streamline that extends to $x \rightarrow-\infty$. Using (81) and (45) we find

$$
F^{\prime}=-\left(\rho_{\infty} U_{\infty}^{2} L M^{2} q / 2 \gamma\right)\left[u_{0}^{2}(\bar{x}, \bar{y})+v_{0}^{2}(\bar{x}, \bar{y})-1\right],
$$

so on using Bernoulli's equation (15)

$$
F^{\prime}=\left(\rho_{\infty} U_{\infty}^{2} L q / \gamma^{2}\right)\left\{\left[p^{\prime}(\bar{x}, \bar{y}) / p_{\infty}\right]-1\right\} .
$$

Thus the impulse opposed to the free stream increases when heat is added at a point where the gas is compressed by the flow and vice versa. In the case of flow of a uniform stream past a fixed cylinder this will lead to either a thrust or a drag, respectively.

There is also now net vorticity $\Gamma$ in the flow given by

$$
\Gamma=\int_{\tilde{\phi}}^{\infty} \int_{\bar{\psi} . \epsilon \epsilon}^{\bar{\psi}+\epsilon} \frac{\omega_{1}(\phi, \psi)}{J(\phi, \psi)} d \phi d \psi
$$

where $\omega_{1}(\phi, \psi)$ is given in Eq. (45). Evaluation of the $\psi$ integral is straightfoward and leads to

$$
\Gamma=\frac{q J(\bar{\phi}, \bar{\psi})}{2 \gamma} \int_{\bar{\phi}}^{\infty} \frac{1}{J^{2}} \frac{\partial J}{\partial \psi} d \phi .
$$

If $\alpha$ is the inclination to the $x$ axis of the streamline $\bar{\psi}$ through the source, then

$$
\Gamma=\frac{q}{\gamma}\left[u_{0}^{2}(\bar{x}, \bar{y})+v_{0}^{2}(\bar{x}, \bar{y})\right] \int_{\bar{\phi}}^{\infty} \frac{1}{J} \frac{\partial \alpha}{\partial \phi} d \phi .
$$

The associated lift acting on the cylinder is $G^{\prime}$, where

$$
G^{\prime}=\rho_{\propto} U_{\infty}^{2} L M^{2} \Gamma .
$$

The lift vanishes for a symmetric flow when the source is on the downstream axis of symmetry, for then $\alpha=0$. We note that for flow past a cylinder (86) and (89) and (90) can be verified using a contour integration of the Lagally ${ }^{11}$ type.

\section{DISCUSSION}

We have examined the influence of a steady heat source on a slighly compressible fluid using a generalized RayleighJanzen expansion, with the aim of determining the $O\left(M^{2}\right)$ interaction between the heat release and the compressible fluid motion. The heat source produces a sheetlike entropy layer confined to that portion of the unperturbed flow streamline downstream of the source position. This in turn generates an $O\left(M^{2}\right)$ singular vorticity wake in the form of coincident vortex and dipole sheets, from which the pertur- bation to the incompressible flow caused by the presence of the heat source can be calculated.

The special case of a heat source located on the downstream axis of symmetry of a self-propagating vortex pair has been analyzed in detail. In this flow the vortex wake consists of a dipole vortex sheet only, and when combined with the flow perturbation caused by compressibility alone, an expansion of the vortex pair and an $O\left(M^{2}\right)$ increase in the pair impulse results. An analysis of the mass-momentum balance for the pair motion reveals that this increase in pair impulse, and also an accompanying outward mass flux at infinity is provided by a balancing wake influx of momentum and mass from infinitely far downstream along the trailing stagnation streamline. The result is that the pair slows down while expanding laterally, and the flow is unsteady. In our case the rate of increase of impulse is $O\left(M^{2}\right)$ while the unsteadiness is $O\left(M^{4}\right)$. We might in fact anticipate acoustic radiation, but a detailed calculation of the $O\left(M^{4}\right)$ velocity field for the perturbed vortex pair motion would be difficult.

To calculate the vortex pair expansion from (64) requires that we propose an ansatz for the position and strength of the point heat release in relation to the zerothorder incompressible flow. Rather than attempting to model a real combustion process we choose a simple illustrative example by fixing the dimensionless heat release of the point heat source $q$, and its dimensionless position $\bar{x}$ as constants. We further define the dimensionless scaling by fixing the vortex circulation $\Gamma$ and the free-stream sound speed $C_{\infty}$. We note that in the parametrically unsteady flow there is no mechanism for changing $\Gamma$ since there is no heat release in the recirculation region. From (1) and the definition of $M$ following (10) the product $U_{\infty} M^{2}$ then scales as $L^{-3}$, and we may write, using (61) and (64)

$$
\frac{d L}{d t}=\frac{q\left(\bar{x}^{2}-1\right) \Gamma^{3}}{128 \pi^{4} \gamma\left(\bar{x}^{2}+1\right)^{2} C_{\infty}^{2}} \frac{1}{L^{3}},
$$

thus giving, for large time, a $t^{1 / 4}$ power law growth in the vortex separation. We note that different growth profiles result from different choices for the physical parameters but the result is always a slow growth of $2 L$ with time.

For a general flow we find that momentum is added to the flow if heat is released in regions where the gas is compressed. In the case of flow past a rigid body this gives rise to a thrust, while the net vorticity created yields a lift.

The boundaries of these regions can be easily determined from (86) and (7), (13), and (15) together with $u_{0}(x, y)$ and $v_{0}(x, y)$ for the incompressible flow. For the vortex pair flow it may thus be shown that the pair impulse increases if heat is added at points $(\bar{x}, \bar{y})$ on those streamlines exterior to $\mathbf{R}$ where

$$
\bar{x}^{2}>1+\bar{y}^{2},
$$

As the vortex pair separation slowly increases, as, for example, is given by (91), then the bounding bubble $\mathbf{R}$ may be expected to undergo a Lagrangian deformation, and it is expected that this will eventually have a profound effect on the combustion problem which-in a crude way-we are attempting to model. A self-consistent calculation of the bubble deformation would require a systematic treatment of the 
$O\left(M^{2}\right)$ perturbation to the incompressible flow due to separate contributions from the parametric unsteadiness (i.e., the pair expansion), the direct heat release interaction [Eq. (57) ] and from the effect of pure flow compressibility, as given in MP. While specific calculations have not been attempted we would expect generally that heat release should lead to a slow enlargement of $\mathbf{R}$ that will entrain oxidizer. In this case, for large time, at least some of the heat release will then take place inside $\mathbf{R}$, and our analysis will fail.

\section{ACKNOWLEDGMENTS}

We are grateful to Dr. E. G. Broadbent and Dr. J. E. Broadwell for helpful discussion and for comments on the manuscript.
This work was supported by a Science and Engineering Research Council grant.

'A. R. Karagozian and B. V. S. Manda, Combust. Sci. Technol. 49, 185 (1986).

${ }^{2}$ P. A. McMurtry, W.-H. Jou, J. J. Riley, and R. W. Metcalf, AIAA J. 24, 962 (1986).

${ }^{3}$ E. G. Broadbent, Ing. Arch, 40, 24 (1971).

${ }^{4}$ D. R. Chenoweth, Q. Appl. Math. 22, 177 (1964).

${ }^{5}$ D. W. Moore and D. I. Pullin, J. Fluid Mech. 185, 171 (1987).

${ }^{6} \mathrm{R}$. Kronig and J. C. Brink, Appl. Sci. Res. Sec. A. 2, 142 (1950).

${ }^{7}$ A. S. Brignell, Int. J. Heat 18,61 (1975).

${ }^{8}$ E. G. Broadbent, Prog. Aerosp. Sci. 17, 93 (1976).

${ }^{9}$ A. Barsony-Nagy, J. Er-El, and S. Yungster, J. Fluid Mech. 178, 367 (1987).

${ }^{10}$ L. C. Woods, Theory of Subsonic Plane Flow (Cambridge U.P., London, 1961).

"L. M. Milne-Thomson, Theoretical Hydrodynamics (Macmillan, New York, 1968), 5th ed., p. 224. 